\title{
Impact of engineering parameters on performance of relay-assisted network
}

\author{
Issam Maaz ${ }^{1}$, Jean-Marc Conrat ${ }^{2}$, Jean-Christophe Cousin ${ }^{3}$, Samer Alabed ${ }^{4}$ \\ ${ }^{1,4}$ College of Engineering and Technology, American University of the Middle East, Kuwait \\ ${ }^{2}$ Wireless Engineering Propagation, Orange Labs, France \\ ${ }^{3}$ Communications and Electronics Department, Telecom ParisTech, France
}

\begin{tabular}{l}
\hline Article Info \\
\hline Article history: \\
Received Jan 22, 2020 \\
Revised Mar 15, 2020 \\
Accepted Apr 11, 2020 \\
\hline
\end{tabular}

\section{Keywords:}

Capacity

Cooperative communications

Distributed systems

Measurment campaign

Small cells

\begin{abstract}
This paper compares the performance of a relay assisted network to the performance given by a classical macrocell network without the presence of relay node schemes. The capacity enhancement provided by a relaying system as a function of the relay antenna height and the propagation environment surrounding the relay nodes is analyzed and discussed in details. The analysis in this work is based on the theoretical Shannon capacity where both measured/experimental path loss and calibrated path loss models are taken into consideration. In this work, we assume a decode and forward scheme, a full-duplex relaying protocol and an optimized relay location is investigated. A $30 \%$ of improvement in the macrocell capacity is achieved with the usage of relaying scenario compared to a classical macrocell network. Furthermore, increasing the relay antenna height from 4 meters to 12 meters can significantly increase the relay capacity to more than $20 \%$ in suburban and moderate urban environments.
\end{abstract}

Copyright (C) 2020 Institute of Advanced Engineering and Science. All rights reserved.

\section{Corresponding Author:}

Samer Alabed,

Department of Electrical Engineering,

College of Engineering and Technology,

American University of the Middle East,

P.O.Box: 220 Dasman, 15453, Kuwait

Email: Samer.Al-abed@aum.edu.kw

\section{INTRODUCTION}

Enhanced capacity and extended coverage with increased cell edge bit rates are among the desired requirements of the beyond $5 \mathrm{G}$ wireless communication systems. Many solutions are being proposed in order to meet the above requirements. Among them, the relay technology appears to be a promising solution [1]. A basic relay network is composed of a Base Station (BS), Relay Station (RS) and Mobile Station (MS). The RS is an intermediate node which receives, processes and forwards the signal between BS and MS. By assuming that the backhaul link (BS-RS link) and the access link (RS-MS) attenuate less the signal that the direct link (BS-MS link) can do, one can expect a throughput gain compared to the direct transmission when the signal is transferred via the relay.

Many studies have investigated the performance of the relaying technique either with a theoretical approach [2], or with system-level simulations [3] or with field measurements [4]. Moreover, many recent multiple antenna techniques have been proposed to improve the overall system performance in terms of bit error rate, the achievable data rate [5-12] and system complexity [13-15] such as space time coding techniques [5-6], beamforming techniques [7-9], time reversal technique [12], relay selection techniques [16-28], and differential techniques [6-9]. However, there are still lacks on understanding the impact of the propagation conditions on the performance of the relaying technique. There is no clear answer if it makes sense from a strictly propagation point of view to transmit directly a signal from the source to the destination 
or to transmit the signal via a relay. Furthermore, according to the authors' knowledge the impact of key engineering parameters such as the relay antenna height or the relay position have not been considered yet.

This paper aims to compare the relay-assisted communication throughput with the throughput provided by the direct link. Path loss models or measured values used for the analysis are extracted from a measurement campaign that was described in [29-32]. Reference [29] is focused on the backhaul link whereas [30-32] are focused on the access link. All publications gave many details about the measurement scenario and the BS, RS and MS locations. The comparison between relay-assisted and direct communication is performed for different relay antenna heights and positions. The paper is divided into four main chapters: Section 2 describes the proposed method, Section 3 explains the research method, results are provided in Section 4 and the conclusion is drawn in Section 5.

\section{PROPOSED METHOD}

We consider a base station (BS) that tries to communicate with the mobile by the assistance of a fixed relay. Both RS and MS are deployed in an outdoor environment and hence no penetration loss is taken into account. BS, RS and MS are alone in the cell and there is no interference from the other communicating nodes. These simulation assumptions would be oversimplified to estimate realistic throughputs but they are well-adapted in order to understand the impacts of the propagation channel or deployment parameters on the throughput. Table 1. summarizes the simulation parameters adopted which are in agreement with [33]. Two scenarios are defined. The first one is illustrated in Figure 1. where the BS, RS and MS are collinear. This scenario is used to quantify the impact of the relay antenna or the relaying protocol.

Table 1. Simulation parameters

\begin{tabular}{cc}
\hline Variable & Value \\
\hline BS transmit power & $49 \mathrm{dBm}$ \\
RS transmit power & $33 \mathrm{dBm}$ \\
Bandwidth, B & $20 \mathrm{MHz}$ \\
Carrier frequency & $2.2 \mathrm{GHz}$ \\
RS antenna height, $h_{r s}$ & $5-10 \mathrm{~m}$ \\
MS antenna height & $2 \mathrm{~m}$ \\
Tooftop level, hroof & $15-25 \mathrm{~m}$ \\
Path loss, PL & Measured path loss or modeled one \\
\hline
\end{tabular}

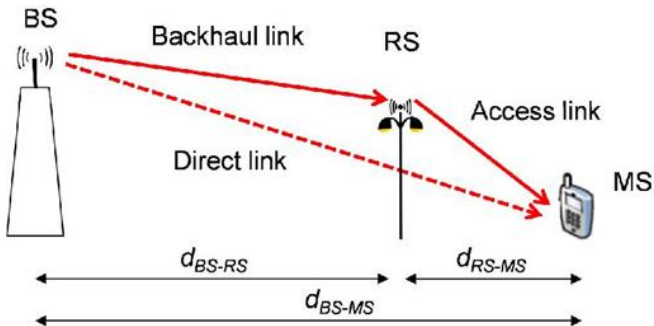

Figure 1. Relaying scenario

PL is calculated with models described in [29-32] and summarized by (1-6). Since RS and MS are always under the rooftops in an urban environment, the optical visibility between BS and RS or between BS and MS is unlikely. Therefore, we consider a non line of sight (NLOS) single slope PL model for the direct and backhaul links. Regarding the access link, we consider a dual slope PL model which is a combination between the line of sight (LOS) and NLOS components since MS may have a LOS connection with RS when MS is close to RS. The LOS/NLOS distance dependent probability is defined in [32]. Received powers are processed by assuming isotropic antenna with $0 \mathrm{dBi}$ gain at RS, BS and MS.

$$
\begin{aligned}
& P L_{B S-M S}=33.4 \log _{10}\left(d_{B S-M S}\right)+38.7 \\
& P L_{B S-R S}=33.4 \log _{10}\left(d_{B S-M S}\right)+38.7 \\
& \quad-20 \log _{10}\left(h_{\text {roof }}-2\right)+20 \log _{10}\left(h_{\text {roof }}-h_{R S}\right) \\
& P L_{L O S}(d B)=20 \log _{10}\left(d_{R S-M S}\right)+39 \\
& P L_{N L O S}(d B)=46 \log _{10}\left(d_{R S-M S}\right)+14.2-0.6 h_{R S} \\
& P L_{R S-M S}=p_{L r o b_{L O S}} P L_{L O S}+\left(1-p r o b_{L O S}\right) P L_{N L O S} \\
& \operatorname{prob}_{L O S}\left(d_{R S-M S}\right)=0.5-\min \left(0.5,5 \exp \left(-156 / d_{R S-M S}\right)\right) \\
& \quad+\min \left(0.5,5 \exp \left(-d_{R S-M S} / 30\right)\right)
\end{aligned}
$$


with $d_{B S-M S}$, the distance between BS and MS and $d_{R S-M S}$ the distance between RS and MS. The second scenario is a measurement replay scenario where BS, RS and MS locations or path loss values are conducted from the measurement campaign. This scenario is used to illustrate the impact of the relay location.

\section{RESEARCH METHOD}

We define $C_{B S-M S}, C_{B S-R S}$ and $C_{R S-M S}$ as capacities related to link BS-MS, BS-RS and RS-MS respectively. The capacity is calculated using Shannon's equation:

$$
C=B \log (1+S N R)
$$

with SNR, the signal to noise ratio. We consider three relaying protocol: half-duplex (HD), modified half duplex (mHD) and the full-duplex (FD). The decode-and-forward scheme was selected as it has been shown that amplify-and-forward outperforms decode-and-forward protocol especially for low SNR, which is the case when the mobile is located at the cell edge [34]. The capacity in relay-assisted communication can be computed according to the relaying protocol.

a) HF relaying: In this relaying protocol, the resources are shared between the access and backhaul link in the time or frequency domain in order to avoid self-interference. Figure 2 illustrates a HF relay with time sharing. The capacity experienced at the MS is given as follows:

$$
C_{H D}=(1 / 2) \min \left(C_{B S-R S}, C_{R S-M S}\right)
$$

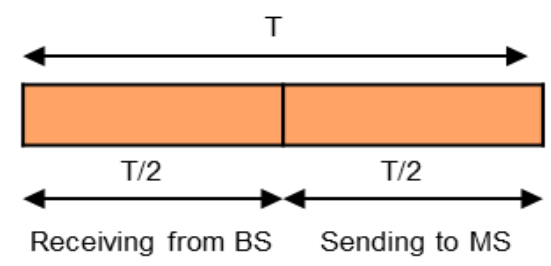

Figure 2. HD relay protocol

b) m-HD relaying: According to (8), the capacity can be maximized if $C_{B S-R S}$ and $C_{R S-M S}$ are equal. The mHD relaying protocol allocates $\alpha$ and 1- $\alpha$ fraction of time or frequency band for the links BS-RS and RS-MS respectively as shown in Figure 3. Thus, the maximum capacity can be obtained when $\alpha C_{B S-R S}=(1-\alpha) C_{R S-M S}$ and $C_{m H D}$ can be written as follows:

$$
\mathcal{C}_{m H D=}\left(c_{B S R S} \mathcal{C}_{R S}\right) /\left(c_{B S R S}{ }^{+} \mathcal{C}_{R S M S}\right)
$$

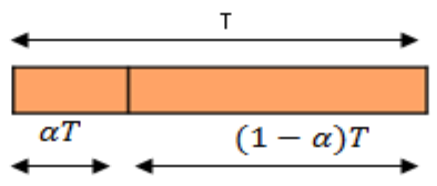

Figure 3. mHD relay protocol

c) FD relaying: RS simultaneously receives the signal from BS and forwards it to MS at the same timefrequency radio resource as shown in Figure 4 and the capacity can be calculated as follows:

$$
C_{F D}=\min \left(C_{B S-R S}, C_{R S-M S}\right)
$$




\section{$\mathrm{T}$}

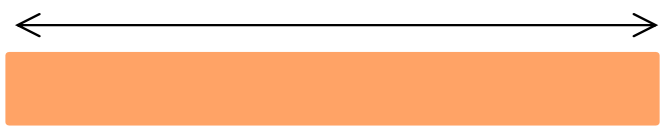

simultaneously receiving from

BS and sending towards MS

Figure 4. Full duplex protocol

This relaying protocol doubles the channel capacity compared to the HD protocol. However, this capacity can be achieved if self-interference at RS can be completely canceled. Several methods have been proposed in order to mitigate the self-interference [35]. The FD protocol also assumes advanced receiver able to handle simultaneously the signal coming from BS and RS.

\section{RESULTS AND DISCUSSION}

Simulation and experimental results based on different relaying schemes, relay antenna height and relay positions are presented in this Section.

\subsection{Relaying protocol}

The macrocell inter-site distance in urban environment ranged approximately between $500 \mathrm{~m}$ and $1500 \mathrm{~m}$ depending on the urban human density. Therefore, the distance BS-RS may range between 250 $\mathrm{m}$ and $750 \mathrm{~m}$ assuming a BS-RS distance equal to the half of the interdistance. Figure 5(a) and Figure 5(b) shows the relay capacity as a function of the different relaying protocol for a BS-RS distance equal to $300 \mathrm{~m}$ and $600 \mathrm{~m}$. The relay capacity achieves a maximum for all protocols when the mobile is close to RS and is symmetrical compared to a vertical axis set at a distance equal to the distance BS-RS. $C_{F D}$ is constant for short $d_{R S-M S}$ due to the limitation of $C_{B S-R S}$. In the example given by Figure 5, this limitation occured for $d_{B S-M S}$ ranged from $200 \mathrm{~m}$ to $400 \mathrm{~m}$. If RS was at street level, the threshold would be $C_{B S-M S}$ at $d_{B S-R S}$. As RS is located above the street level, the threshold is shifted upwards compared to the direct link capacity and the threshold level depends on the antenna height. When MS gets away from RS terminal, $C_{F D}$ is no more limited by the backhaul link but by the access link and $C_{F D}$ is equal to $C_{R S-M S}$. The capacity difference between the macrolink and the relay link depends on $d_{B S-R S}$, but $\mathrm{HD}$ and $\mathrm{m}-\mathrm{HD}$ capacities are almost systematically lower than the direct link capacity. The bad performance of these relaying protocols is due to the time-frequency resource sharing between the access and backhaul links. Practically, carrier aggregation techniques or specific scheduling algorithm favoring cell edge users would give higher performances than $\mathrm{HD}$ and $\mathrm{m}-\mathrm{HD}$ relay and would not require any additional equipment deployment. Hence, $\mathrm{HD}$ or $\mathrm{m}-\mathrm{HD}$ relay is not an efficient solution to diminish the cell edge problem. The FD relay gives a substantial gain compared to the $\mathrm{HD}$ or m-HD relay. In the example given in Figure 5, the FD relay outperforms the direct link for $d_{B S \text { - }}$ ${ }_{M S}$ ranged from $200 \mathrm{~m}$ to $500 \mathrm{~m}$.

The full-duplex capacity is strongly limited by the backhaul capacity and would not be an efficient solution compared to a microcell with a fiber optics backhaul. However, fiber optics may be not accessible in an entire city and $C_{B S-R S}$ may be increased by increasing the antenna height, by optimizing the relay location or by using directive antenna with higher gain at RS. Hence, the full duplex relay is a potential solution for enhancing the channel capacity. In the following section, we consider only the FD protocol and define a new metric to compare simply the relay-assisted communication throughput with the direct throughput as defined in the following (11):

$$
C_{\text {ratio }}=100 \times \frac{C_{F D}-C_{B S-M S}}{C_{B S-M S}}
$$

For $C_{\text {Ratio }}<0$, the direct link is better than the relay-assisted one. When $C_{\text {Ratio }}>0$, the relaying solution increases the capacity compared to the direct link. The relay coverage refers to the geographical area where $C_{\text {Ratio }}$ is positive. 


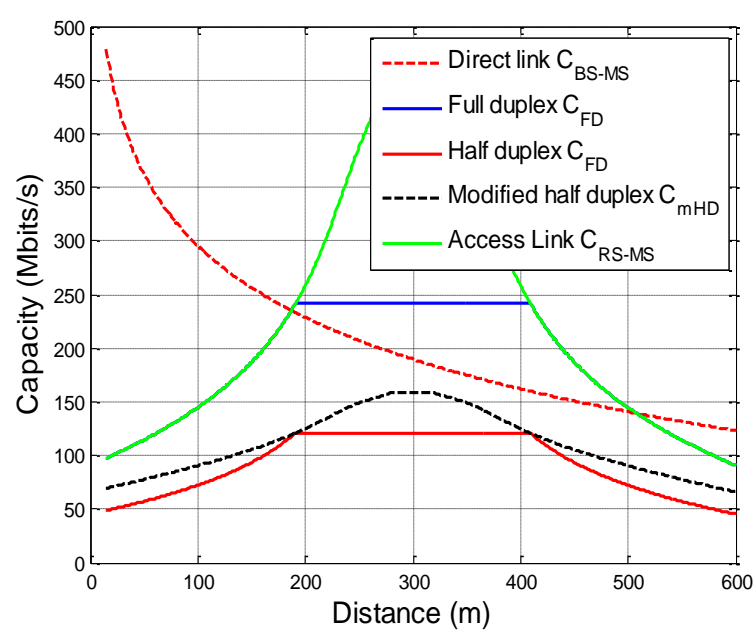

(a)

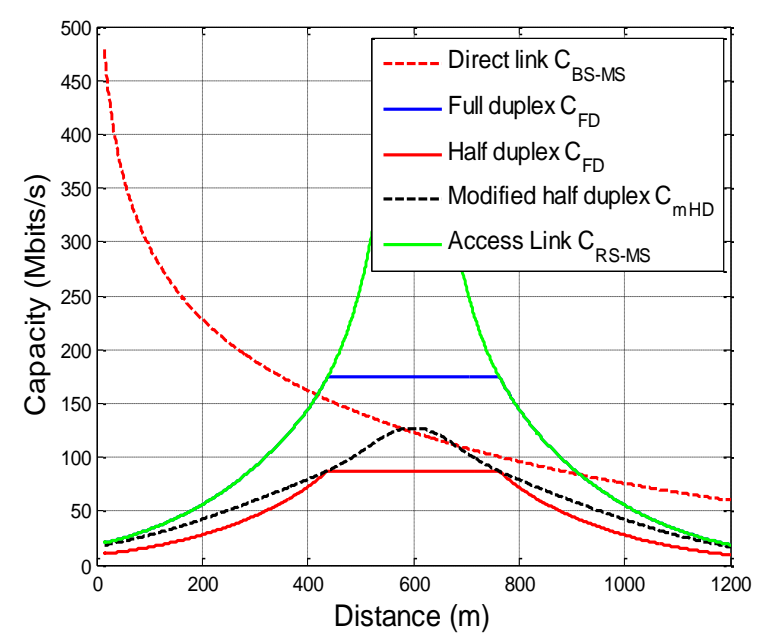

(b)

Figure 5. (a) Capacity as function of relaying protocol, $h_{\text {roof }}=15 \mathrm{~m}, h_{r s}=10 \mathrm{~m}, d_{B S-R S}=300 \mathrm{~m}$

(b) Capacity as function of relaying protocol, $h_{\text {roof }}=15 \mathrm{~m}, h_{r s}=10 \mathrm{~m}, d_{B S-R S}=600 \mathrm{~m}$

\subsection{Relay antenna height}

Figure 6(a) and 6(b) show $C_{F D}$ and $C_{\text {Ratio }}$ for two different relay antenna heights (5 and 10 meters) and two different rooftop levels (15 and 25 meters). We observe that $C_{\text {Ratio }}$ ranges from $0 \%$ and $40 \%$ depending on $d_{B S-R S}, h_{\text {roof }}$ and $h_{r s}$. The higher the antenna height, the higher the capacity ratio. However, this relation is not linear especially when the relay capacity is limited by the backhaul capacity. As indicated by eq. 2, the backhaul PL is not a linear function of the relay distance to the ground but an exponential function of the relay distance to the building rooftop level. With $h_{\text {roof }}=25 \mathrm{~m}, C_{\text {Ratio }}$ increases by about $20 \%$ between the lowest and highest antenna height. With $h_{\text {roof }}=15 \mathrm{~m}, C_{\text {Ratio }}$ increases by about $20 \%$ between the lowest and highest antenna height. This result suggests that the relay antenna height would have a significant impact in suburban environment or moderate urban environment when buildings have 3 or 4 floors, but will have a limited impact in dense urban environment when buildings have more than 5 floors.

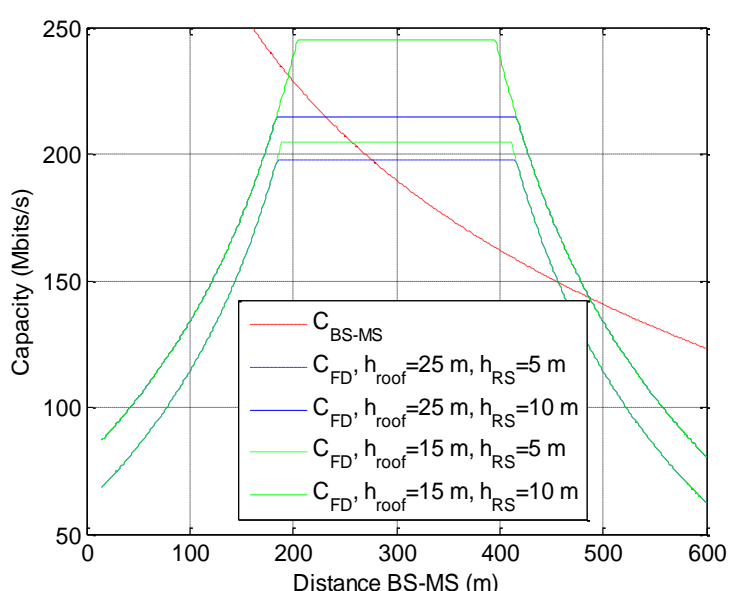

(a)

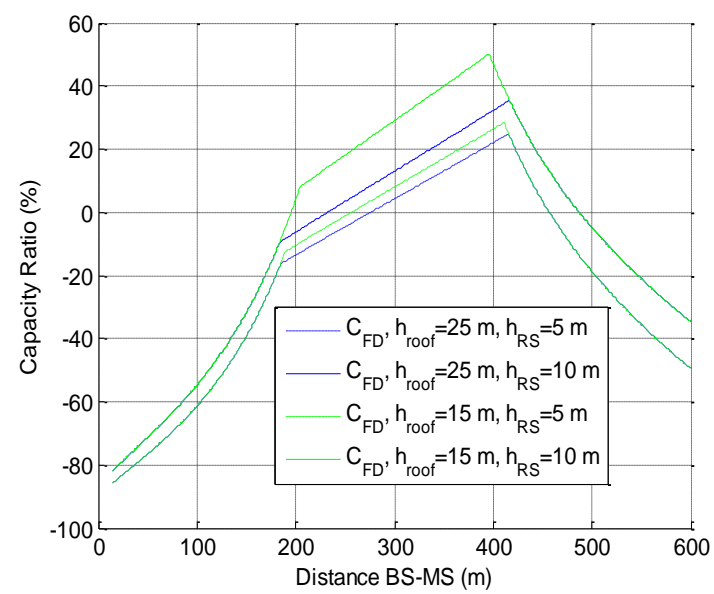

(b)

Figure 6. (a) Impact on antenna height on relay capacity (b) Capacity ratio as function of the antenna height

\subsection{Relay location}

The performance of the relaying technique is also affected by the relay position, i.e. by the environment around the relay as illustrated by Figures 7 and 8. In order to highlight areas where the relay provides gain compared to the macrocell, $3 C_{\text {Ratio }}$ levels are arbitrary defined: $C_{\text {Ratio }}<0 \%, 0 \%<C_{\text {Ratio }}<30 \%$ 
and $C_{\text {Ratio }}>30 \%$. Figure 7(a) and 7(b) show $C_{\text {Ratio }}$ of RS9 and RS7 respectively. RS7 is located in a street canyon perpendicular to the BS-RS axis and RS9 is located beside a park with moderate vegetation. We observe clearly that the RS9 coverage is better than that of RS7 although RS7 and RS9 were located very close to each other. Different distances between BS and RS, or between RS and MS cannot explain the difference in the performance. Therefore the environment around the relay may change significantly $C_{F D}$. We also observe that RS7 and RS9 coverage are complimentary. An optimal relay location would be somewhere between R7 and RS9, probably at the nearest street intersection.

Figure 8(a) and 8(b) give two other examples illustrating the impact of the relay position. RS1 and RS2 are still close to each other, but RS1 is located at a street intersection and RS2 is located in a small square surrounded by buildings. The RS2 coverage is limited by its position while RS1 is located in a small segment of the main road, whereas RS1 coverage includes most the RS2 coverage as well it includes many streets with a ratio capacity higher than $30 \%$ and higher than $50 \%$ in limited segments. Both examples demonstrate that relay performances can be enhanced by an optimizing the relay location. Practically, an efficient relay deployment would require powerful planning tool software including consistent and comprehensive propagation channel models for macrocell and microcell environments.

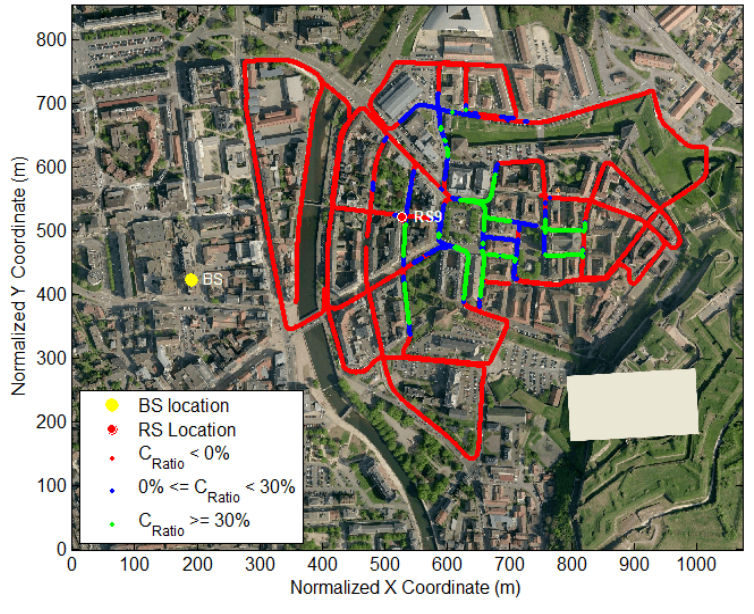

(a)

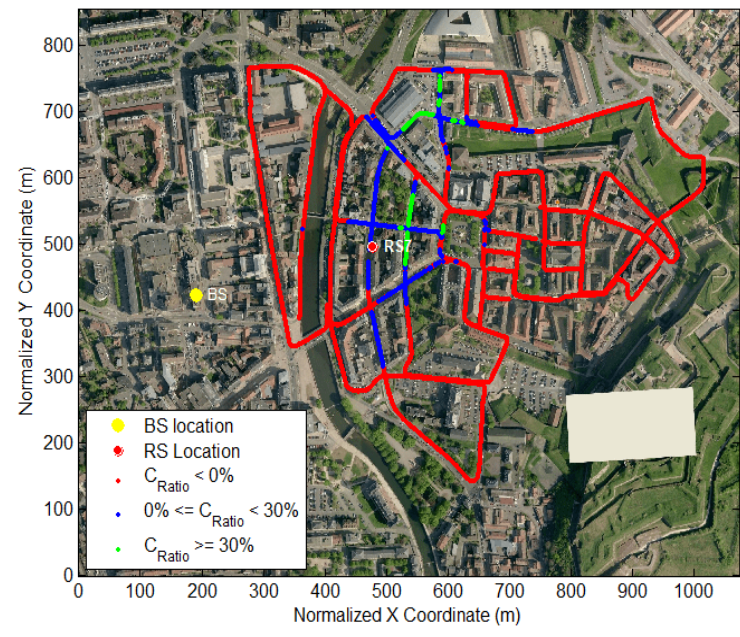

(b)

Figure 7. (a) Capacity ratio RS9, $h_{R S}=8.8 \mathrm{~m}$ (b) Capacity ratio $\mathrm{RS} 7, h_{R S}=8.8 \mathrm{~m}$

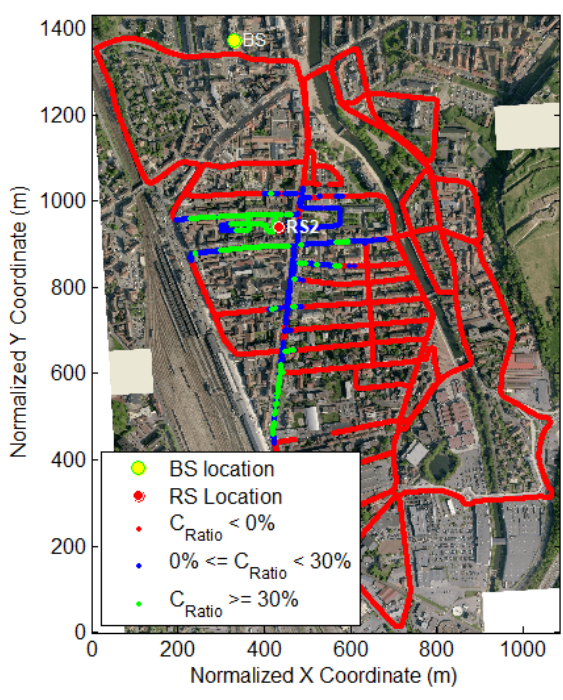

(a)

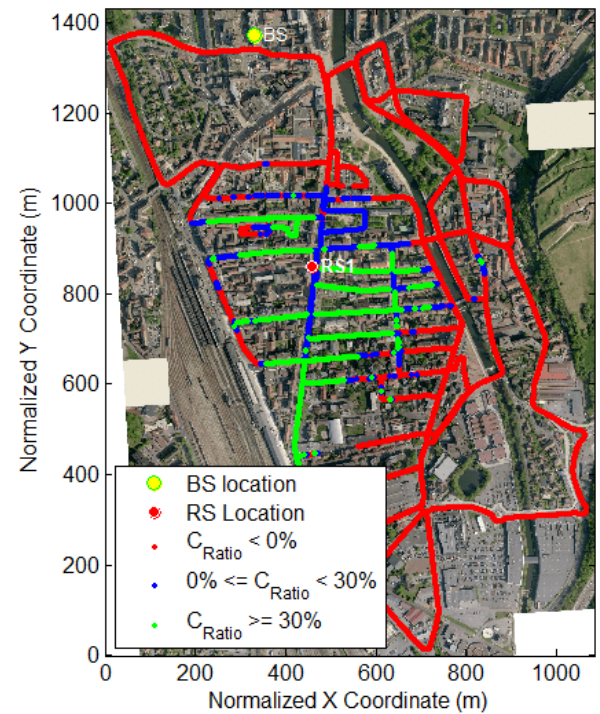

(b)

Figure 8. (a) Capacity ratio $\mathrm{RS} 2, h_{R S}=8.8 \mathrm{~m}$ (b) Capacity ratio $\mathrm{RS} 1, h_{R S}=8.8 \mathrm{~m}$ 


\section{CONCLUSION}

We showed that the direct link capacity can be overperformed by a relay-assisted communication when a full-duplex relay technology is used. The capacity gain provided by the relay reaches more than $30 \%$ depending on the antenna relay height and location. The analysis assumed omnidirectional antenna at RS and was performed at $2.2 \mathrm{GHz}$. Future work will analyze the frequency dependency of relay performances. Higher frequencies would allow the use of antenna array with a limited physical size but able to generate high gain directional beam enhancing the backhaul and access link capacities. But the actual benefits of directional antennas depend on the received signal spatial distribution. Consequently, future work will also investigate the directions of arrival at RS.

\section{REFERENCES}

[1] A. Papadogiannis, et al, "Pass it on: Advanced relaying concepts and challenges for networks beyond 4G," IEEE Vehicular Technology Magazine, vol. 9, no. 2, pp. 29-37, 2014.

[2] G. Weisi and T. O'Farrell, "Relay deployment in cellular networks: Planning and optimization," IEEE Selected Areas in Communications, vol. 31, no. 8, pp. 1597-1606, 2013.

[3] A. Bou Saleh, et al, "On the coverage extension and capacity enhancement of inband relay deployments in LTEAdvanced networks," Journal of Electrical and Computer Engineering, vol. 2010, pp. 1-12, 2010.

[4] Q. Yu, H. Asplund, J. E. Berg, and G. Zhiheng, "Non-Line-of-Sight 2.6GHz relay backhaul channel performance: field test and analysis," in 2012 IEEE Vehicular Technology Conference (VTC Fall), pp. 1-5, 2012.

[5] S. Alabed, J. Paredes, and A. B. Gershman, "A simple distributed space-time coded strategy for two-way relaychannels," IEEE Transactions on Wireless Communications, vol. 11, no. 4, pp. 1260-1265, 2012.

[6] S. Alabed, M. Pesavento, and A. Klein, "Non-coherent distributed space-time coding techniques for two-way wireless relay networks,” Signal Processing, vol. 93, no. 12, pp. 3371-3381, 2013.

[7] S. Alabed and M. Pesavento, "A simple distributed differential transmit beamforming technique for two-way wireless relay networks," In 2012 International ITG Workshop on Smart Antennas (WSA), pp. 243-247, 2012.

[8] A. Schad, et al, "Bi-directional differential beamforming formulti-antenna relaying," 2015 IEEE International Conference on Acoustics, Speech and Signal Processing (ICASSP), pp. 2884-2888, 2015.

[9] S. Alabed, "Performance analysis of differential beamforming in decentralized networks," International Journal of Electrical and Computer Engineering, vol, 8, no. 3, pp. 1692-1700, 2018.

[10] S. Alabed, "Computationally efficient multi-antenna techniques for multi-user two-way wireless relay networks," International Journal of Electrical and Computer Engineering, pp. 1684-1691, vol. 8, no. 32018.

[11] S. Alabed, J. Paredes, A. Gershman, "A low complexity decoder for quasi-orthogonal space-time block codes," IEEE Transactions on Wireless Communication, vol. 3, no. 10, pp. 988-994, 2011.

[12] T. Dubois, M. Hélard, M. Crussière, I. Maaz, “Time reversal applied to large MISO-OFDM systems," 2013 IEEE 24th International Symposium on Personal Indoor and Mobile Radio Communications (PIMRC), pp. 896-901, 2013.

[13] Nasaruddin, et al, "Optimized power allocation for cooperative amplify-and-forward with convolutional codes," Indonesian Journal of Electrical Engineering and Computer Science, vol. 12, no. 8, pp. 6243-6253, 2014.

[14] Kehinde Odeyemi and Erastus Ogunti, "Capacity enhancement for high data rate wireless communication system," International Journal of Electrical and Computer Engineering, vol. 4, no. 5, pp. 800-809, 2014.

[15] Nasaruddin, Yunida, Khairul Munadi, "Improved model of the selection with soft and hard combining decoding strategies for multi-user multi-relay cooperative networks," International Journal of Electrical and Computer Engineering (IJECE), vol. 6, no. 4, pp. 1766-1778, 2016.

[16] S. Alabed, M. Pesavento, A. Klein, "Relay selection based space-time coding for two-way wireless relay networks using digital network coding," ISWCS 2013; The Tenth International Symposium on Wireless Communication Systems, pp. 1-5, 2013.

[17] Y. Li, R. Louie, and B. Vucetic, "Relay selection with network coding in two-way relay channels," IEEE Transactions on Vehicular Technology, vol. 59, no. 9, pp. 4489-4499, 2010.

[18] G. Chen, Z. Tian, Y. Gong, Z. Chen, and J. Chambers, "Max-ratio relay selection in secure buffer-aided cooperative wireless networks," IEEE Transactions on Information Forensics and Security, vol. 9, no. 4, pp. 719-729, 2014.

[19] S. Atapattu, Y. Jing, H. Jiang, C. Tellambura, "Relay selection schemes and performance analysis approximations for two-way networks," IEEE Trans. Commun, vol. 61, no. 3, pp. 987-998, 2013.

[20] S. Alabed, "Performance analysis of two-way DF relay selection techniques," ICT Express, vol. 2, no. 3, pp. 91-95, 2016.

[21] S. Alabed, "Performance analysis of bi-directional relay selection strategy for wireless cooperative communications," EURASIP Journal on Wireless Communications and Net., vol. 2019, no. 97, pp. 1-11, 2019.

[22] G. Cai, Y. Fang, G. Han, J. Xu, G. Chen, "Design and analysis of relay-selection strategies for two-way relay network-coded DCSK systems," IEEE Trans. Veh. Technol, vol. 67, no. 2, pp. 1258-1271, 2018.

[23] T. Nguyen, et al, "Performance enhancement for energy harvesting based two-way relay protocols in wireless adhoc networks with partial and full relay selection methods," Ad Hoc Netw, vol. 84, pp. 178-187, 2019.

[24] S. Zhou, J. Xu, Z. Niu, "Interference-aware relay selection technique for two-hop relay networks with multiple source-destination pairs," IEEE Trans. Veh. Technol, vol. 62, no. 5, pp. 2327-2338, 2013.

[25] M. Ju, K. Hwang, H. Song, "Relay selection of cooperative diversity networks with interference-limited destination," IEEE Trans. Veh. Technol, vol. 62, no. 9, pp. 4658-4665, 2013. 
[26] Y. Fang, G. Han, P. Chen, F. C. M. Lau, G. Chen, L. Wang, "A survey on DCSK-based communication systems and their application to UWB scenarios," IEEE Communication Surveys Tuts, vol. 18, no. 3, pp. 1804-1837, 2016.

[27] G. Cai, Y. Fang, G. Han, "Design of an adaptive multiresolution M-ary DCSK system," IEEE Communication Letters, vol. 21, no. 1, pp. 60-63, 2017.

[28] G. Cai, Y. Fang, G. Han, F. C. M. Lau, L. Wang, "A square constellation-based M-ary DCSK communication system," IEEE Access, vol. 4, pp. 6295-6303, 2016.

[29] J. M. Conrat, et al, "Path loss model comparison for LTE-Advanced relay backhaul link in urban environment," The 8th European Conference on Antennas and Propagation (EuCAP 2014), pp. 3472-3476, 2014.

[30] I. Maaz, J. M. Conrat, and J. C. Cousin, "Path loss models in LOS conditions for relay mobile channels," The 8th European Conference on Antennas and Propagation (EuCAP 2014), pp. 3475-3479, 2014.

[31] I. Maaz, J. M. Conrat, and J. C. Cousin, "Path loss models in NLOS conditions for relay mobile channels," 2014 IEEE 80th Vehicular Technology Conference (VTC2014-Fall), pp. 1-5, 2014.

[32] I. Maaz, J. M. Conrat, and J. C. Cousin, "Channel model validation for the relay-mobile link in microcell environment," 2015 IEEE 82nd Vehicular Technology Conference (VTC2015-Fall), pp. 1-5, 2015.

[33] 3GPP, "Further advancements for E-UTRA physical layer aspects," vol. TR 36.814 V9.0.0, March 2010, 2010. [Online] Available: http://www.3GPP.org

[34] A. B. Saleh, et al, "Performance of amplify-and-forward and decode-and-forward relays in LTE-advanced," 2009 IEEE 70th Vehicular Technology Conference Fall, pp. 1-5, 2009.

[35] L. Gang, F. R. Yu, J. Hong, V. C. M. Leung, and L. Xi, "In-Band Full-Duplex Relaying: A Survey, Research Issues and Challenges," IEEE Communications Surveys \& Tutoria, vol. 17, no. 2, pp. 500-524, 2015. 